\title{
Reinduction Chemotherapy with Gemtuzumab Ozogamicin and Intermediate/High-Dose Cytarabine: A Single-Center Experience
}

\author{
Sohsuke Meshitsuka1, Mitsuo Hori², Shoichi Mitsuhashi' ${ }^{1}$, Yasushi Okoshi'2,3*, \\ Hiroshi Kojima1 \\ ${ }^{1}$ Department of Clinical Oncology, Ibaraki Prefectural Central Hospital, Ibaraki, Japan \\ ${ }^{2}$ Department of Hematology, Ibaraki Prefectural Central Hospital, Ibaraki, Japan \\ ${ }^{3}$ Ibaraki Clinical Education and Training Center, University of Tsukuba Hospital, Ibaraki, Japan \\ Email: yokoshi@md.tsukuba.ac.jp
}

Received 11 January 2014; revised 11 February 2014; accepted 18 February 2014

Copyright (C) 2014 by authors and Scientific Research Publishing Inc.

This work is licensed under the Creative Commons Attribution International License (CC BY). http://creativecommons.org/licenses/by/4.0/

(c) (i) Open Access

\begin{abstract}
We performed a retrospective analysis of 9 patients with acute myeloid leukemia (AML) treated with gemtuzumab ozogamicin (GO) plus cytarabine as a salvage regimen (GO reinduction) for patients who did not achieve complete remission (CR) after the first cycle of induction chemotherapy or at first relapse. Cases of AML secondary to myelodysplastic syndrome or myeloproliferative disorder were included. CR was achieved in 6 of 9 patients, and 2 of 6 responders became longterm survivors. No non-responders survived longer than 6 months. Toxicity was mild, and the median duration of myelosuppression was less than 30 days. Stomatitis, nausea and sepsis occurred as non-hematological adverse events. Although our sample size was too small to permit definitive conclusions, GO reinduction should be considered for patients who relapse or do not achieve CR after the first cycle of induction chemotherapy. Some AML subtypes may respond more robustly than others, and further investigation is warranted.
\end{abstract}

\section{Keywords}

Gemtuzumab Ozogamicin; AML; Reinduction; Salvage

\footnotetext{
*Corresponding author.
} 


\section{Introduction}

The mainstay of induction chemotherapy for acute myeloid leukemia (AML), cytarabine (AraC) and anthracycline, achieves remission in $70 \%$ - 80\% of untreated patients younger than 60 years of age [1] [2]. Most patients, however, eventually relapse, and the 5-year overall survival rate for this group is as low as 40\% - 45\% [3] [4]. In elderly patients ( $\geq 60$ years old), the remission rate is less than $50 \%$ and the long-term leukemia-free survival rate is less than $10 \%[4]$.

In general, patients who do not achieve complete remission (CR) after the first cycle of induction chemotherapy are retreated with their original regimen or with AraC and another anthracycline. Although long-term outcomes are similar for patients who require 1 versus 2 cycles of induction therapy to achieve CR [5], whether or not the patient achieves CR is critical.

Therapeutic strategies for patients with relapsed AML are limited, but a small percentage of these patients do achieve remission by salvage or reinduction chemotherapy.

Gemtuzumab ozogamicin (GO) is a humanized anti-CD33 monoclonal antibody conjugated to a highly potent antitumor antibiotic, calicheamicin, which induces double-stranded DNA breaks, cell cycle arrest, and apoptosis. GO was originally approved in the United States for the treatment of elderly patients with AML after relapse [6] $[7]$.

In 2008, Chevallier et al. reported long-term disease-free survival after GO, intermediate-dose AraC and mitoxantrone in patients with CD33-positive primary resistant or relapsed AML [8]. The role of combination chemotherapy regimens consisting of GO, AraC and anthracycline for the second cycle of induction therapy or for relapsed AML should be further investigated because the efficacy of conventional chemotherapies for these patients is limited.

We treated patients with GO plus AraC with or without anthracycline at Ibaraki Prefectural Central Hospital, Japan from 2009 to 2011. Here, we report the treatment outcomes of these patients and discuss the safety and efficacy of GO with reference to recent data.

\section{Patients and Methods}

We performed a retrospective analysis of adult patients who were treated with GO as salvage induction chemotherapy from March 2009 to August 2011 at Ibaraki Prefectural Central Hospital, Ibaraki, Japan. AML was diagnosed at initial presentation based on the World Health Organization (Fourth Edition) and French-AmericanBritish classification systems. This study included both de novo AML as well as AML secondary to myelodysplastic syndrome (MDS) or myeloproliferative disorder. Cytogenetic risk groups were defined according to the Bordeaux Grenoble Marseille Toulouse (BGMT) group criteria [9]. Initial induction chemotherapy in the majority of patients consisted of the “ $3+7$ regimen," i.e., idarubicin (12 mg/m² days 1 - 3) and AraC (100 mg/m²/day days 1 - 7). When complete remission was achieved, 3 courses of consolidation therapy consisting mainly of high-dose AraC plus anthracycline were planned.

Salvage induction chemotherapy including GO (hereafter referred to as "GO reinduction") was initiated for patients who did not achieve CR after the first cycle of induction chemotherapy or for patients at first relapse. Relapse was defined as loss of CR.

\subsection{GO Reinduction}

Nine patients were treated with GO reinduction (Table 1), which consisted of mainly high-dose AraC and GO with or without anthracyclines. Six received a remission induction regimen consisting of AraC $1000 \mathrm{mg} / \mathrm{m}^{2}$ twice a day via 3-hour intravenous infusion (iv) from days 1 - 5, mitoxantrone $12 \mathrm{mg} / \mathrm{m}^{2} /$ day via 30-minute iv from days 1 - 3, and GO $6 \mathrm{mg} / \mathrm{m}^{2}$ via 2-hour iv on day 4 or 7 . One patient received idarubicin $6 \mathrm{mg} / \mathrm{m}^{2}$ on days 1 3 instead of mitoxantrone, and 2 received a regimen without anthracyclines. These modifications were determined by the doctors in charge for each patient. All patients met certain criteria, including 1) confirmation of CD33-positive AML by immunophenotyping, 2) serum creatinine and bilirubin levels $<2$ mg/dL, and 3) adequate cardiac function (ejection fraction $>50 \%$ ).

All patients received standard supportive care, including prophylactic and therapeutic antibiotics, as well as transfusion of blood products to maintain platelet counts above $20 \times 10^{9} / \mathrm{L}$. A serotonin receptor antagonist and prednisolone $40 \mathrm{mg} /$ day were administered daily from days 1 - 5 for antiemesis and prophylaxis of adverse reac- 
Table 1. Patient characteristics.

\begin{tabular}{|c|c|c|c|c|c|c|c|c|c|c|c|}
\hline \multirow{2}{*}{ 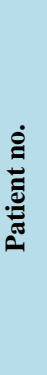 } & \multirow{2}{*}{ 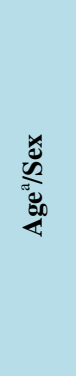 } & \multirow[b]{2}{*}{ 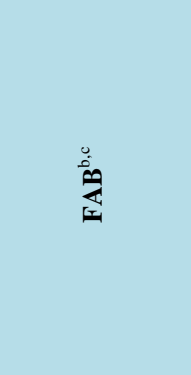 } & \multirow{2}{*}{ 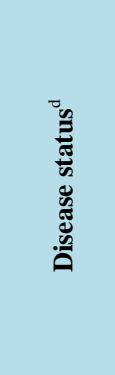 } & \multirow{2}{*}{ 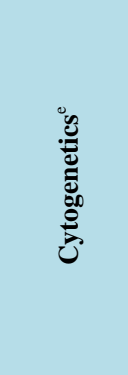 } & \multirow{2}{*}{ 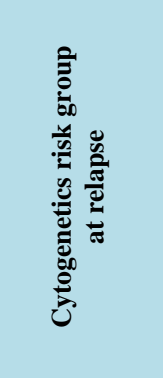 } & \multirow{2}{*}{ 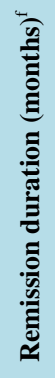 } & \multicolumn{2}{|r|}{ 号 苞 } & \multirow{2}{*}{\multicolumn{2}{|c|}{ 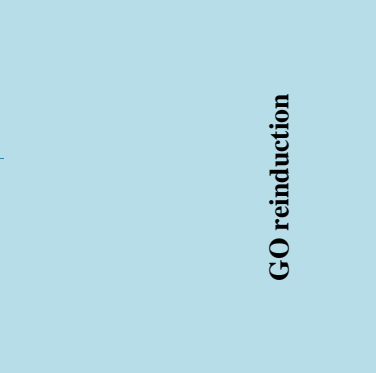 }} & \\
\hline & & & & & & & 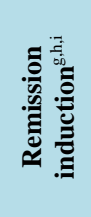 & 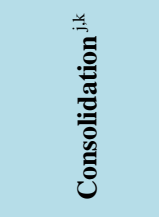 & & & \\
\hline 1 & $65 / M$ & M6 & Non-CR & $\begin{array}{c}\text { complex } \\
\text { karyotype }\end{array}$ & Unfavorable & * & $\begin{array}{l}\text { IDA + } \\
\text { AraC }\end{array}$ & - & $\begin{array}{c}\text { AraC } 2000 \\
\mathrm{mg} / \mathrm{m}^{2} \\
\text { on days } 1 \text { - } 5\end{array}$ & $\begin{array}{c}+ \text { MIT } 12 \mathrm{mg} / \mathrm{m}^{2} \\
\text { on days } 1-3\end{array}$ & $\begin{array}{c}\text { +GO } 6 \mathrm{mg} / \mathrm{m}^{2} \\
\text { on day } 4\end{array}$ \\
\hline 2 & $19 / \mathrm{F}$ & M1 & Non-CR & 46, XX & Intermediate & * & $\begin{array}{l}\text { IDA + } \\
\text { AraC }\end{array}$ & - & $\begin{array}{c}\text { AraC } 2000 \\
\mathrm{mg} / \mathrm{m}^{2} \\
\text { on days } 1 \text { - } 5\end{array}$ & $\begin{array}{l}+ \text { MIT } 12 \mathrm{mg} / \mathrm{m}^{2} \\
\text { on days } 1-3\end{array}$ & $\begin{array}{c}\text { +GO } 6 \mathrm{mg} / \mathrm{m}^{2} \\
\text { on day } 4\end{array}$ \\
\hline 3 & $81 / \mathrm{M}$ & $\begin{array}{l}\text { MDS (RA) } \\
\Rightarrow \text { M2 }\end{array}$ & Non-CR & 46, XY & Intermediate & * & $\begin{array}{l}\text { IDA + } \\
\text { BHAC }\end{array}$ & - & $\begin{array}{c}\text { AraC } 2000 \\
\mathrm{mg} / \mathrm{m}^{2} \\
\text { on days } 1 \text { - } 5\end{array}$ & $\begin{array}{c}+ \text { MIT } 12 \mathrm{mg} / \mathrm{m}^{2} \\
\text { on days } 1-3\end{array}$ & $\begin{array}{c}+\mathrm{GO} 6 \mathrm{mg} / \mathrm{m}^{2} \\
\text { on day } 4\end{array}$ \\
\hline 4 & $56 / F$ & M1 & Non-CR & $\begin{array}{c}\text { Not } \\
\text { Available }\end{array}$ & $\begin{array}{c}\text { Not } \\
\text { Available }\end{array}$ & * & $\begin{array}{l}\text { IDA + } \\
\text { AraC }\end{array}$ & - & $\begin{array}{l}\text { AraC } 2000 \\
\mathrm{mg} / \mathrm{m}^{2} \\
\text { on days } 1 \text { - } 5\end{array}$ & $\begin{array}{l}+ \text { MIT } 12 \mathrm{mg} / \mathrm{m}^{2} \\
\text { on days } 1-3\end{array}$ & $\begin{array}{c}\text { +GO } 6 \mathrm{mg} / \mathrm{m}^{2} \\
\text { on day } 7\end{array}$ \\
\hline 5 & 73/M & M2 & $\begin{array}{l}\text { First } \\
\text { relapse }\end{array}$ & 46, XY & Intermediate & 5 & CAG & $\begin{array}{l}\text { IDA + AraC, } \\
\text { BHAC-DM }\end{array}$ & $\begin{array}{c}\text { AraC } 100 \\
\mathrm{mg} / \mathrm{m}^{2} \\
\text { on days } 1-5\end{array}$ & & $\begin{array}{c}+\mathrm{GO} 6 \mathrm{mg} / \mathrm{m}^{2} \\
\text { on day } 6\end{array}$ \\
\hline 6 & $71 / \mathrm{M}$ & $\begin{array}{l}\text { MDS (RA) } \\
\Rightarrow \text { M2 }\end{array}$ & $\begin{array}{l}\text { First } \\
\text { relapse }\end{array}$ & $\begin{array}{c}\text { complex } \\
\text { karyotype }\end{array}$ & Unfavorable & 10 & $\begin{array}{l}\text { IDA + } \\
\text { AraC }\end{array}$ & $\begin{array}{c}\text { HDAraC + } \\
\text { MIT, ×3 }\end{array}$ & $\begin{array}{c}\text { AraC } 2000 \\
\mathrm{mg} / \mathrm{m}^{2} \text { on days } \\
1-5\end{array}$ & & $\begin{array}{c}+\mathrm{GO} 6 \mathrm{mg} / \mathrm{m}^{2} \\
\text { on day } 6\end{array}$ \\
\hline 7 & $42 / \mathrm{M}$ & M0 & $\begin{array}{l}\text { First } \\
\text { relapse }\end{array}$ & 46, XY & Intermediate & 19 & $\begin{array}{l}\text { IDA + } \\
\text { AraC }\end{array}$ & $\begin{array}{c}\text { HDAraC + } \\
\text { MIT, } \times 2\end{array}$ & $\begin{array}{c}\text { AraC } 2000 \\
\mathrm{mg} / \mathrm{m}^{2} \\
\text { on days } 1 \text { - } 5\end{array}$ & $\begin{array}{c}+ \text { MIT } 12 \mathrm{mg} / \mathrm{m}^{2} \\
\text { on days } 1-3\end{array}$ & $\begin{array}{c}+\mathrm{GO} 6 \mathrm{mg} / \mathrm{m}^{2} \\
\text { on day } 7\end{array}$ \\
\hline 8 & $67 / M$ & M0 & $\begin{array}{c}\text { First } \\
\text { relapse }\end{array}$ & 46, XY & Intermediate & 4 & $\begin{array}{l}\text { IDA + } \\
\text { AraC }\end{array}$ & $\begin{array}{l}\text { HDAraC + } \\
\text { MIT, } \times 3\end{array}$ & $\begin{array}{l}\text { AraC } 2000 \\
\mathrm{mg} / \mathrm{m}^{2} \\
\text { on days } 1 \text { - } 5\end{array}$ & $\begin{array}{c}+ \text { IDA } 6 \mathrm{mg} / \mathrm{m}^{2} \\
\text { on days } 1-3\end{array}$ & $\begin{array}{c}+\mathrm{GO} 6 \mathrm{mg} / \mathrm{m}^{2} \\
\text { on day } 7\end{array}$ \\
\hline 9 & $62 / M$ & $\begin{array}{l}\text { Essential } \\
\text { thrombocythemia } \\
\Rightarrow \mathrm{M} 2\end{array}$ & $\begin{array}{l}\text { First } \\
\text { relapse }\end{array}$ & 46, XY & Intermediate & 2 & $\begin{array}{l}\text { IDA + } \\
\text { AraC }\end{array}$ & $\begin{array}{c}\text { HDAraC + } \\
\text { MIT, ×1 }\end{array}$ & $\begin{array}{c}\text { AraC } 2000 \\
\mathrm{mg} / \mathrm{m}^{2} \\
\text { on days } 1 \text { - } 5\end{array}$ & $\begin{array}{c}+ \text { MIT } 12 \mathrm{mg} / \mathrm{m}^{2} \\
\text { on days } 1-3\end{array}$ & $\begin{array}{c}+\mathrm{GO} 6 \mathrm{mg} / \mathrm{m}^{2} \\
\text { on day } 4\end{array}$ \\
\hline
\end{tabular}

AraC, cytarabine; IDA, idarubicin; GO, gemtuzumab ozogamicin; MIT, mitoxantrone. ${ }^{a}$ Age at the initiation of GO therapy. ${ }^{b}$ FAB (French-AmericanBritish) subtype at first presentation (not at the initiation of GO therapy). "MDS (RA) $\Rightarrow$ M2" indicates AML M2 secondary to MDS refractory anemia according to the FAB classification. "“"Non-CR” indicates a patient who did not achieve complete remission after the first cycle of induction

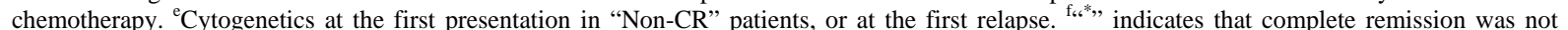
achieved before GO therapy. ${ }^{g}$ IDA + AraC: IDA $12 \mathrm{mg} / \mathrm{m}^{2}$ on days $1-3$ and AraC $100 \mathrm{mg} / \mathrm{m}^{2}$ on days 1 - 7. ${ }^{\mathrm{h}}$ IDA + BHAC: IDA $12 \mathrm{mg} / \mathrm{m}^{2}$ on days 1 - 3 and enocitabine $200 \mathrm{mg} / \mathrm{m}^{2}$ on days $1-7$. ${ }^{\mathrm{i}} \mathrm{CAG}$ : AraC $10 \mathrm{mg} / \mathrm{m}^{2}$ on days $1-14$, aclarubicin hydrochloride $14 \mathrm{mg} / \mathrm{m}^{2}$ on days $1-4$, and filgrastim 200 $\mu \mathrm{g} / \mathrm{m}^{2}$ on days $1-14$. ${ }^{\mathrm{B}} \mathrm{BHAC}-\mathrm{DM}$ : enocitabine $170 \mathrm{mg} / \mathrm{m}^{2}$ on days $1-5$, daunomycin $30 \mathrm{mg} / \mathrm{m}^{2}$ on days 1 and 4 , and mercaptopurine hydrate 70 $\mathrm{mg} / \mathrm{m}^{2}$ on days $1-7$.

tions to AraC. Two medications were administered prior to GO: hydroxyzine pamoate $25 \mathrm{mg}$ and hydrocortisone sodium succinate $100 \mathrm{mg}$. Continuous intravenous heparin was used for prophylaxis of veno-occlusive disease (VOD) for 2 patients (Nos. 8 and 9). Ursodeoxycholic acid or monoammonium glycyrrhizinate were only used if clinically indicated. During therapy, patients were monitored by routine blood tests, including complete blood cell count, coagulation test, blood chemistry, and weekly assessment of serum 1, 3-beta-D-glucan levels.

\subsection{Response Definitions}

CR was defined using conventional criteria: morphologic leukemia-free state, defined by $<5 \%$ blasts in a bone 
marrow aspirate sample; absence of blasts with Auer rods; absence of extramedullary leukemia; absolute neutrophil count $\geq 1.0 \times 10^{9} / \mathrm{L}$; platelet count $\geq 100 \times 10^{9} / \mathrm{L}$; and red blood cell transfusion independence. Remission duration was measured from the date of CR to the date of relapse or death from any cause. Patients without events were censored at the date of the last follow-up. Overall survival was measured from the initiation of GO reinduction to the date of death from any cause. Survival curve was calculated by Kaplan-Meier survival analysis using Microsoft Excel.

\subsection{Toxicity}

Toxicities during GO reinduction were evaluated using the Common Terminology Criteria for Adverse Events (CTCAE), version 4 (http://ctep.cancer.gov/protocolDevelopment/electronic_applications/ctc.htm).

\section{Results}

\subsection{Treatment Outcomes}

Patient characteristics are summarized in Table 1. Four patients did not achieve CR after the first cycle of induction chemotherapy; 5 were in the first relapse after achieving CR. Two patients (Nos. 3 and 6) had AML secondary to MDS, and 1 (No. 9) had AML transformed from essential thrombocythemia. In the relapsed patients, the median duration of remission before GO reinduction was 5 months (range, 2 - 19 months).

Response to GO reinduction and outcomes are shown in Table 2. CR was achieved in 6 of 9 patients, with a median time to CR of 31 days (range, 27 - 36 days). Six responders received further therapy; of these, 2 patients remained in CR (Nos. 2, 7), 3 experienced relapse (Nos. 4, 6, 8), and 1 died suddenly of non-treatment-related aspiration during CR (No. 9). Of the 3 non-responders, 1 received further treatment but died of disease progression, 1 died of sepsis, and 1 did not receive further treatment because of poor performance status. Five of the 6 patients who achieved CR, but none of the non-responders, survived for more than 6 months. Median overall survival of 9 patients was 284 days (range, 15 - 724) (Figure 1).

\subsection{Toxicities}

Toxicities are summarized in Table 3. Overall, the GO reinduction was well tolerated. Mild (CTCAE grades 0 2) nausea, headache and fever, but no coagulopathy, were observed during administration of GO and other drugs. The median durations of neutropenia (defined as neutrophil count $<0.5 \times 10^{9} / \mathrm{L}$ ) and thrombocytopenia (platelet count $<50 \times 10^{9} / \mathrm{L}$ and platelet transfusion dependence) were 25 and 27 days, respectively.

One individual (patient No. 3, an 81 year-old male) died of sepsis during myelosuppression. None of the patients in this study developed severe (CTCAE grade 3 or more) hepatic toxicity or VOD.

\section{Discussion}

Chevallier et al. reported the outcomes of a single-arm study for primary resistant or relapsed AML treated by GO $9 \mathrm{mg} / \mathrm{m}^{2}$ on day 4 , AraC $1 \mathrm{~g} / \mathrm{m}^{2}$ every 12 hours on days $1-5$, and mitoxantrone $12 \mathrm{mg} / \mathrm{m}^{2}$ on days $1-3$ [8]. Of 62 patients, 18 were refractory and 44 relapsed; rates of CR and CR with delayed platelet recovery were $50 \%$ and $13 \%$, respectively. The overall, event-free and disease-free survival rates were $41 \%, 33 \%$ and $53 \%$ at 2 years, respectively. Previous attempts to combine the approved dose of GO $\left(9 \mathrm{mg} / \mathrm{m}^{2}\right)$ with chemotherapy, however, resulted in excess toxicities, particularly liver toxicity [10]. Thus, we chose a GO dose of $6 \mathrm{mg} / \mathrm{m}^{2}$ in our reinduction regimen.

In 2011, Prebet et al. reported treatment outcome with GO and AraC for AML in the first relapse [11]. In this retrospective analysis, 34 patients were treated with intermediate- to high-dose AraC and GO (average dose 6 $\mathrm{mg} / \mathrm{m}^{2} /$ day, range 3 - 9) $+/$ - other cytotoxic drugs; 21 patients (58\%) were with AraC $2000 \mathrm{mg} / \mathrm{m}^{2} /$ day on days 1 5 , GO, and mitoxantrone $12 \mathrm{mg} / \mathrm{m}^{2} /$ day on days $1-3$. Overall response rate was $68 \%$. Overall and event-free survival were 35 and 24 months, respectively. Comparing other 56 AML patients treated with intermediate- to high-dose AraC containing regimen without GO, improved outcome was shown in low- and intermediate-risk cytogenetics groups, although patients treated with GO were more frequently transplanted with allogeneic hematopoietic stem cell.

Recently, GO was voluntarily withdrawn from the United States market after the randomized SWOG 0106 
Table 2. Treatment outcome.

\begin{tabular}{|c|c|c|c|c|c|c|c|}
\hline $\begin{array}{l}\text { Patient } \\
\text { no. }\end{array}$ & $\operatorname{Age}^{\mathrm{a}} / \operatorname{Sex}$ & $\begin{array}{l}\text { Disease } \\
\text { status }^{b}\end{array}$ & $\begin{array}{l}\text { Days to } \\
\text { CR }^{\mathrm{C}}\end{array}$ & $\begin{array}{c}\text { Successive treatments } \\
\text { after GO } \\
\text { reinduction }\end{array}$ & $\begin{array}{l}\text { Remission duration } \\
\text { after GO reinduction } \\
\text { (days) }\end{array}$ & $\begin{array}{l}\text { Overall } \\
\text { survival } \\
\text { (days) }\end{array}$ & Outcome \\
\hline 1 & $65 / \mathrm{M}$ & Non-CR & * & $\begin{array}{c}\text { FLAG-IDA, } \\
\text { HDAraC + DNR }\end{array}$ & * & 99 & $\begin{array}{l}\text { Died of disease } \\
\text { progression }\end{array}$ \\
\hline 2 & $19 / F$ & Non-CR & 30 & HDAraC + MIT & $\geq 694$ & $\geq 724$ & Alive in CR \\
\hline 3 & 81/M & Non-CR & $*$ & - & $*$ & 15 & Died of sepsis \\
\hline 4 & $56 / F$ & Non-CR & 27 & $\begin{array}{c}\text { HDAraC + MIT, } \\
\text { HDAraC + DNR, } \\
\text { FLAG-IDA, } \\
\text { HDAraC + GO }\end{array}$ & 257 & 284 & Died of sepsis \\
\hline 5 & $73 / \mathrm{M}$ & First relapse & * & - & * & 116 & $\begin{array}{l}\text { Died of disease } \\
\text { progression }\end{array}$ \\
\hline 6 & 71/M & First relapse & 33 & CAG, AZA & 139 & 344 & $\begin{array}{c}\text { Died of disease } \\
\text { progression }\end{array}$ \\
\hline 7 & $42 / \mathrm{M}$ & First relapse & 36 & HDAraC + MIT, $\times 2$ & $\geq 173$ & $\geq 209$ & Alive in CR \\
\hline 8 & $67 / \mathrm{M}$ & First relapse & 27 & FLAG + MIT, ×3 & 219 & 312 & $\begin{array}{c}\text { Died of disease } \\
\text { progression }\end{array}$ \\
\hline 9 & $62 / \mathrm{M}$ & First relapse & 32 & $\mathrm{GO}+\mathrm{iAraC}+\mathrm{MIT}$ & 59 & 91 & Died of aspiration \\
\hline
\end{tabular}

AraC, cytarabine; CR, complete remission; DNR, daunomycin; GO, gemtuzumab ozogamicin; HDAraC, high dose cytarabine; iAraC, intermediate dose cytarabine; IDA, idarubicin; MIT, mitoxantrone. "Age at the initiation of GO therapy. "'Non-CR" indicates a patient who did not achieve complete remission after the first cycle of induction chemotherapy. ${ }^{\mathrm{c}}$ “”” $^{*}$ indicates that complete remission was not achieved. ${ }^{\mathrm{d}}$ FLAG-IDA: AraC 2000 $\mathrm{mg} / \mathrm{m}^{2}$ on days $1-5$, fludarabine $30 \mathrm{mg} / \mathrm{m}^{2}$ on days $1-5$, and IDA $8 \mathrm{mg} / \mathrm{m}^{2}$ on days $1-3$. ${ }^{\mathrm{e}} \mathrm{HDAraC}+\mathrm{DNR}$ : AraC $4500 \mathrm{mg} / \mathrm{m}^{2}$ on days $1-3 \mathrm{and}$ DNR $45 \mathrm{mg} / \mathrm{m}^{2}$ on days 1 - 3. ${ }^{\mathrm{f}} \mathrm{HDAraC}+\mathrm{MIT}$ : AraC $4000 \mathrm{mg} / \mathrm{m}^{2}$ on days $1-4$ and MIT $5 \mathrm{mg} / \mathrm{m}^{2}$ on days $1-2$ or on days $1-3$. ${ }^{\mathrm{g}} \mathrm{HDAraC}+\mathrm{GO}$ : AraC $4000 \mathrm{mg} / \mathrm{m}^{2}$ on days $1-5$ and GO $6 \mathrm{mg} / \mathrm{m}^{2}$ on day 8 . ${ }^{\mathrm{h}} \mathrm{CAG}$ : AraC $10 \mathrm{mg} / \mathrm{m}^{2}$ on days 1 - 14 , aclarubicin hydrochloride $14 \mathrm{mg} / \mathrm{m}^{2}$ on days $1-4$, and filgrastim $200 \mu \mathrm{g} / \mathrm{m}^{2}$ on days $1-14$. ${ }^{\mathrm{i}}$ AZA: azacitidine $80 \mathrm{mg} / \mathrm{m}^{2}$ on days $1-7$. ${ }^{\mathrm{j}} \mathrm{FLAG}+\mathrm{MIT}$ : AraC $2000 \mathrm{mg} / \mathrm{m}^{2}$ on days $1-5$, fludarabine 30 $\mathrm{mg} / \mathrm{m}^{2}$ on days $1-5$, and MIT $7 \mathrm{mg} / \mathrm{m}^{2}$ on days 1 , 3, and 5 . ${ }^{\mathrm{k}} \mathrm{GO}+\mathrm{iAraC}+\mathrm{MIT}$ : AraC $2000 \mathrm{mg} / \mathrm{m}^{2}$ on days $1-5$, MIT $12 \mathrm{mg} / \mathrm{m}^{2}$ on days $1-3$, and GO $6 \mathrm{mg} / \mathrm{m}^{2}$ on day 4 .

\section{Table 3. Adverse events.}

\begin{tabular}{|c|c|c|c|c|c|c|}
\hline \multirow{2}{*}{ Patient no. } & \multirow{2}{*}{ Days to CR } & \multirow{2}{*}{$\begin{array}{l}\text { Duration of } \\
\text { neutropenia } \\
\text { (days) }\end{array}$} & \multirow{2}{*}{$\begin{array}{c}\text { Duration of } \\
\text { thrombocytopenia } \\
\text { (days) }\end{array}$} & \multicolumn{3}{|c|}{ Non-hematological adverse event } \\
\hline & & & & Grade 1 - 2 & Grade 3 - 4 & Grade 5 \\
\hline 1 & $*$ & $*$ & * & $\begin{array}{l}\text { fever, diarrhea, skin disorder, } \\
\text { stomatitis, hepatic disorder }\end{array}$ & febrile neutropenia & \\
\hline 2 & 30 & 30 & 26 & hepatic disorder & febrile neutropenia & \\
\hline 3 & $*$ & $*$ & $*$ & & & sepsis \\
\hline 4 & 27 & 25 & 20 & fever, skin disorder & $\begin{array}{c}\text { febrile neutropenia, stomati- } \\
\text { tis, nausea }\end{array}$ & \\
\hline 5 & $*$ & 22 & $*$ & & febrile neutropenia, stomatitis & \\
\hline 6 & 33 & 29 & 46 & fever, stomatitis, nausea & febrile neutropenia & \\
\hline 7 & 36 & 38 & 41 & $\begin{array}{l}\text { diarrhea, epistaxis, anorexia, } \\
\text { headache }\end{array}$ & febrile neutropenia & \\
\hline 8 & 27 & 18 & 28 & fever, diarrhea, stomatitis & febrile neutropenia & \\
\hline 9 & 32 & 21 & 24 & vomiting & febrile neutropenia, nausea & \\
\hline
\end{tabular}

CR, complete remission; DNR, daunomycin; GO, gemtuzumab ozogamicin; HDAraC, high dose cytarabine; iAraC, intermediate dose cytarabine; MIT, mitoxantrone. *indicates that complete remission or blood cell recovery was not achieved.

study, which added $6 \mathrm{mg} / \mathrm{m}^{2}$ of GO to $3+7$ regimen, found an increase in 30-day mortality uncompensated by improvements in CR or survival [12]. Other recent randomized trials comparing induction chemotherapy with or without GO at lower doses $\left(<9 \mathrm{mg} / \mathrm{m}^{2}\right)$, however, found a benefit for GO in newly diagnosed AML, especially patients with more favorable cytogenetics [13] [14]. We summarize these in Table 4 [12] [15]-[18]. Significant differences in CR rates were not found among any of the reports, however, two of five trials showed significant 


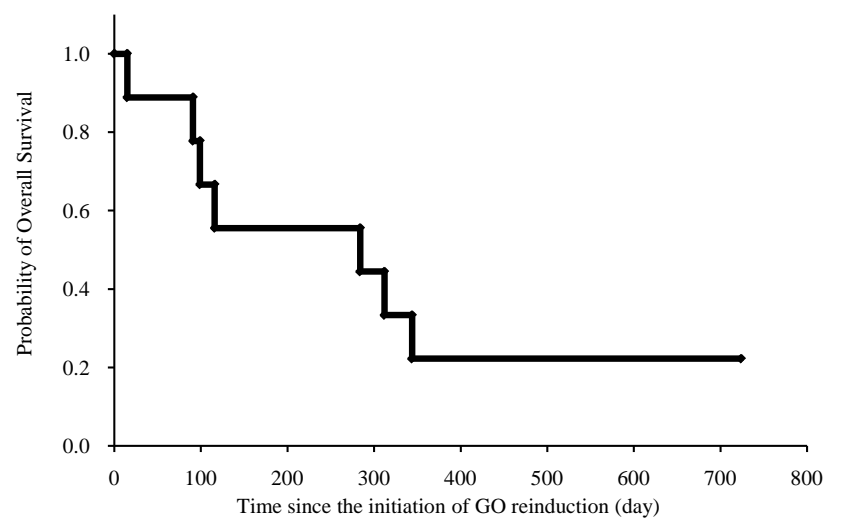

The Kaplan-Meier curve shows overall survival after the initiation of salvage induction chemotherapy including GO (GO reinduction).

Figure 1. Overall survival.

Table 4. Reported randomized trials with or without GO.

\begin{tabular}{|c|c|c|c|c|c|c|c|c|}
\hline Trial & & $\begin{array}{l}\text { No. of } \\
\text { atients }\end{array}$ & & Induction & & utcomes & $\begin{array}{l}\text { Induction } \\
\text { Mortality }\end{array}$ & Trend of a \\
\hline $\begin{array}{l}\text { Author, year } \\
\text { Study name }\end{array}$ & Total & $\begin{array}{c}\text { No GO } v \\
\text { GO }\end{array}$ & $\begin{array}{l}\text { Group } \\
\text { (years) }\end{array}$ & Chemotherapy & $\begin{array}{c}\text { CR Rate } \\
\text { No GO } v \text { GO }\end{array}$ & $\begin{array}{c}\text { Survival } \\
\text { No GO v GO }\end{array}$ & & $\begin{array}{c}\text { GO in } \\
\text { favorable-risk } \\
\text { AML }\end{array}$ \\
\hline $\begin{array}{l}\text { Petersdorf et al., } \\
2009\end{array}$ & 627 & $229 \vee 277$ & $18-60$ & $\begin{array}{c}\text { DNR } 45 \mathrm{mg} / \mathrm{m}^{2} \text { per day on days } \\
1-3+\text { Ara-C } 100 \mathrm{mg} / \mathrm{m}^{2} \text { per } \\
\text { day on days } 1-7+\mathrm{GO} 6 \\
\mathrm{mg} / \mathrm{m}^{2} \text { on day } 4 v \text { DNR } 60 \\
\mathrm{mg} / \mathrm{m}^{2} \text { per day on days } 1-3+ \\
\text { Ara-C } 100 \mathrm{mg} / \mathrm{m}^{2} \text { per day on } \\
\text { days } 1-7\end{array}$ & $69 \%$ v $66 \%^{*}$ & $\begin{array}{l}\text { RFS }^{*} \text { (data not } \\
\text { available) } \\
\text { EFS, NA } \\
\text { median OS, } \\
35 \mathrm{M} v 31 \mathrm{M}^{*}\end{array}$ & $\begin{array}{c}0.8 \% \vee 5.8 \% \\
(p=0.002)\end{array}$ & NA \\
\hline $\begin{array}{l}\text { Burnett et al., } \\
\quad 2011 \\
\text { MRC AML15 }\end{array}$ & 1113 & $557 v 556$ & $<60$ & $\begin{array}{c}\mathrm{DA} v \text { ADE } v \text { FLAG-IDA } \pm \text { GO } \\
3 \mathrm{mg} / \mathrm{m}^{2} \text { on day } 1\end{array}$ & $83 \%$ v $82 \%{ }^{*}$ & $\begin{array}{c}\text { 5-year RFS, } \\
35 \% \text { v 39\% }{ }^{*} \\
\text { EFS, NA } \\
\text { 5-year OS, } \\
41 \% \text { v } 43 \%^{*}\end{array}$ & $6 \% \vee 7 \%{ }^{*}$ & Yes \\
\hline $\begin{array}{l}\text { Castaigne et al., } \\
2012\end{array}$ & 278 & $139 \vee 139$ & $50-70$ & $\begin{array}{l}\text { DNR } 60 \mathrm{mg} / \mathrm{m}^{2} \text { per day on days } \\
\begin{array}{c}1-3+\text { Ara-C } 200 \mathrm{mg} / \mathrm{m}^{2} \text { per } \\
\text { days } 1-7 \pm \mathrm{GO} 3 \mathrm{mg} / \mathrm{m}^{2} \text { per } \\
\text { day on days } 1,4,7\end{array}\end{array}$ & $75 \% v 81 \%^{*}$ & $\begin{array}{c}\text { 2-year RFS, } \\
23 \% \vee 50 \% \\
(p=0.0003) \\
2 \text {-year EFS, } \\
17 \% v 41 \% \\
(p=0.0003) \\
2 \text {-year OS, } \\
42 \% v 53 \% \\
(p=0.037)\end{array}$ & $4 \%$ v $6.5 \%{ }^{*}$ & Yes \\
\hline $\begin{array}{l}\text { Burnett et al., } \\
\qquad 2012 \\
\text { AML16 }\end{array}$ & 1115 & $556 \vee 559$ & $51-84$ & $\begin{array}{l}\text { DNR } 50 \mathrm{mg} / \mathrm{m}^{2} \text { per day on days } \\
1,3,5 \text { and CLO } 20 \mathrm{mg} / \mathrm{m}^{2} \text { per } \\
4 \text { day on days } 1-5 \text { or Ara-C } 100 \\
\mathrm{mg} / \mathrm{m}^{2} \text { every } 12 \text { hours on days } 1 \\
-10 \pm \mathrm{GO} 3 \mathrm{mg} / \mathrm{m}^{2} \text { on day } 1\end{array}$ & $58 \% \vee 62 \%{ }^{*}$ & $\begin{array}{c}\text { 3-year RFS, } \\
16 \% \vee 21 \% \\
(p=0.04) \\
\text { EFS, NA } \\
3 \text {-year OS, } \\
20 \% \vee 25 \% \\
(p=0.05)\end{array}$ & $11 \%$ v $12 \%^{*}$ & Yes \\
\hline $\begin{array}{l}\text { Brunnberg et al., } \\
2012\end{array}$ & 119 & $58 v 57$ & $60-83$ & $\begin{array}{c}\text { Ara-C } 100 \mathrm{mg} / \mathrm{m}^{2} \text { per day on } \\
\text { days } 1-7+\mathrm{DNR} 60 \mathrm{mg} / \mathrm{m}^{2} \text { per } \\
\text { day on days } 1-3 v \text { Ara-C } 100 \\
\mathrm{mg} / \mathrm{m}^{2} \text { per day on days } 1-7+ \\
\mathrm{GO} 6 \mathrm{mg} / \mathrm{m}^{2} \text { on day } 1 \text { and } 4 \\
\mathrm{mg} / \mathrm{m}^{2} \text { on day } 8\end{array}$ & $55 \% v 54 \%^{*}$ & $\begin{array}{c}\text { median RFS, } \\
8 \mathrm{M} v 14 \mathrm{M}^{*} \\
\text { EFS, NA } \\
\text { OS, } 9 \mathrm{M} v 10 \mathrm{M}^{*}\end{array}$ & $\begin{array}{l}5 \% \vee 19 \% \\
(p=0.021)\end{array}$ & NA \\
\hline
\end{tabular}

ADE, cytarabine, daunorubicin, and etoposide; Ara-C, cytarabine; CLO, clofarabine; CR, complete remission; DA, daunorubicin plus cytarabine; DNR, daunorubicin; EFS, event-free survival; FLAG-IDA, fludarabine, cytarabine, granulocyte colony-stimulating factor, and idarubicin; GO, gemtuzumab ozogamicin; M, months; NA, not available; OS, overall survival; RFS, relapse-free survival. " no significant difference. 
increases in overall survival and relapse free survival [16] [17].

Two studies reported higher mortality following a single dose of GO $6 \mathrm{mg} / \mathrm{m}^{2}$ as part of induction therapy [12] [18]. This dosing is identical to our own, and we did in fact experience 1 induction mortality in our sample of 9 patients. Although it is too early to draw definitive conclusions, administration of a single dose of $\mathrm{GO} 6 \mathrm{mg} / \mathrm{m}^{2}$ might result in greater toxicity during induction therapy. A lower dose of GO such as $3 \mathrm{mg} / \mathrm{m}^{2}$ [15] [17], even if it were repeated [16], might be safe, with reduced risk of relapse and improved overall survival [16] [17].

AML is not a homogeneous disease but rather a group of diseases, some of which might be particularly sensitive to GO. Three of 5 trials in Table 4 showed a similar trend of a benefit for GO in favorable-risk AML [15]-[17]. This may indicate that AML varies both genetically and clinically, and GO should be administrated to specific patients.

In conclusion, GO reinduction should be considered for patients who do not achieve CR after the first cycle of induction chemotherapy or who relapse. On the basis of available reports, GO might not improve outcomes in patients at high risk, such as the patients in this study. Further investigations are warranted.

\section{Declaration of Interest}

The authors declare that they have no conflicts of interest.

\section{References}

[1] Fernandez, H.F., Sun, Z., Yao, X., Litzow, M.R., Luger, S.M., Paietta, E.M., Racevskis, J., Dewald, G.W., Ketterling, R.P., Bennett, J.M., Rowe, J.M., Lazarus, H.M. and Tallman, M.S. (2009) Anthracycline Dose Intensification in Acute Myeloid Leukemia. The New England journal of Medicine, 361, 1249-1259. http://dx.doi.org/10.1056/NEJMoa0904544

[2] Mandelli, F., Vignetti, M., Suciu, S., Stasi, R., Petti, M.C., Meloni, G., Muus, P., Marmont, F., Marie, J.P., Labar, B., Thomas, X., Di Raimondo, F., Willemze, R., Liso, V., Ferrara, F., Baila, L., Fazi, P., Zittoun, R., Amadori, S. and de Witte, T. (2009) Daunorubicin versus Mitoxantrone versus Idarubicin as Induction and Consolidation Chemotherapy for Adults with Acute Myeloid Leukemia: The Eortc and Gimema Groups Study aml-10. Journal of Clinical Oncology, 27, 5397-5403.

[3] Burnett, A.K., Hills, R.K., Milligan, D.W., Goldstone, A.H., Prentice, A.G., McMullin, M.F., Duncombe, A., Gibson, B. and Wheatley, K. (2010) Attempts to Optimize Induction and Consolidation Treatment in Acute Myeloid Leukemia: Results of the mrc aml12 Trial. Journal of clinical Oncology, 28, 586-595.

[4] Buchner, T., Berdel, W.E., Haferlach, C., Haferlach, T., Schnittger, S., Muller-Tidow, C., Braess, J., Spiekermann, K., Kienast, J., Staib, P., Gruneisen, A., Kern, W., Reichle, A., Maschmeyer, G., Aul, C., Lengfelder, E., Sauerland, M.C., Heinecke, A., Wormann, B. and Hiddemann, W. (2009) Age-Related Risk Profile and Chemotherapy Dose Response in Acute Myeloid Leukemia: A Study by the German Acute Myeloid Leukemia Cooperative Group. Journal of clinical oncology, 27, 61-69.

[5] Rowe, J.M., Kim, H.T., Cassileth, P.A., Lazarus, H.M., Litzow, M.R., Wiernik, P.H. and Tallman, M.S. (2010) Adult Patients with Acute Myeloid Leukemia who Achieve Complete Remission after 1 or 2 Cycles of Induction Have a Similar Prognosis: A Report on 1980 Patients Registered to 6 Studies Conducted by the Eastern Cooperative Oncology Group. Cancer, 116, 5012-5021. http://dx.doi.org/10.1002/cncr.25263

[6] Sievers, E.L., Larson, R.A., Stadtmauer, E.A., Estey, E., Löwenberg, B., Dombret, H., Karanes, C., Theobald, M., Bennett, J.M., Sherman, M.L., Berger, M.S., Eten, C.B., Loken, M.R., van Dongen, J.J.M., Bernstein, I.D., Appelbaum, F.R. and Appelbaum, F.R. (2001) Efficacy and Safety of Gemtuzumab Ozogamicin in Patients with cd33-Positive Acute Myeloid Leukemia in First Relapse. Journal of Clinical Oncology, 19, 3244-3254.

[7] Larson, R.A., Sievers, E.L., Stadtmauer, E.A., Löwenberg, B., Estey, E.H., Dombret, H., Theobald, M., Voliotis, D., Bennett, J.M., Richie, M., Leopold, L.H., Berger, M.S., Sherman, M.L., Loken, M.R., van Dongen, J.J.M., Bernstein, I.D., Appelbaum, F.R., Mylotarg Study Group (2005) Final Report of the Efficacy and Safety of Gemtuzumab Ozogamicin (Mylotarg) in Patients with cd33-Positive Acute Myeloid Leukemia in First Recurrence. Cancer, 104, $1442-1452$. http://dx.doi.org/10.1002/cncr.21326

[8] Chevallier, P., Delaunay, J., Turlure, P., Pigneux, A., Hunault, M., Garand, R., Guillaume, T., Avet-Loiseau, H., Dmytruk, N., Girault, S., Milpied, N., Ifrah, N., Mohty, M. and Harousseau, J.L. (2008) Long-Term Disease-Free Survival after Gemtuzumab, Intermediate-Dose Cytarabine, and Mitoxantrone in Patients with cd33(+) Primary Resistant or Relapsed Acute Myeloid Leukemia. Journal of Clinical Oncology, 26, 5192-5197.

[9] Dastugue, N., Payen, C., Lafage-Pochitaloff, M., Bernard, P., Leroux, D., Huguet-Rigal, F., Stoppa, A.M., Marit, G., Molina, L., Michallet, M., et al. (1995) Prognostic Significance of Karyotype in de Novo Adult Acute Myeloid Leukemia. The Bgmt Group. Leukemia, 9, 1491-1498. 
[10] Giles, F.J., Kantarjian, H.M., Kornblau, S.M., Thomas, D.A., Garcia-Manero, G., Waddelow, T.A., David, C.L., Phan, A.T., Colburn, D.E., Rashid, A. and Estey, E.H. (2001) Mylotarg (Gemtuzumab Ozogamicin) Therapy Is Associated with Hepatic Venoocclusive Disease in Patients Who Have Not Received Stem Cell Transplantation. Cancer, 92, 406413. http://dx.doi.org/10.1002/1097-0142(20010715)92:2<406::AID-CNCR1336>3.0.CO;2-U

[11] Prebet, T., Etienne, A., Devillier, R., Romeo, E., Charbonnier, A., D’Incan, E., Esterni, B., Arnoulet, C., Blaise, D. and Vey, N. (2011) Improved Outcome of Patients with low- and Intermediate-Risk Cytogenetics Acute Myeloid Leukemia (aml) in First Relapse with Gemtuzumab and Cytarabine versus Cytarabine: Results of a Retrospective Comparative Study. Cancer, 117, 974-981.

http://onlinelibrary.wiley.com/doi/10.1002/cncr.25554/abstract;jsessionid=CC12AA2454773B97BD79855E4376FD6 D.f01t04

[12] Petersdorf, S., Kopecky, K., Stuart, R.K., Larson, R.A., Nevill, T.J., Stenke, L., Slovak, M.L., Tallman, M.S., Willman, C.L., Erba, H. and Appelbaum, F.R. (2009) Preliminary Results of Southwest Oncology Group Study s0106: An International Intergroup Phase 3 Randomized Trial Comparing the Addition of Gemtuzumab Ozogamicin to Standard Induction Therapy versus Standard Induction Therapy Followed by a Second Randomization to Post-Consolidation Gemtuzumab Ozogamicin versus No Additional Therapy for Previously Untreated Acute Myeloid Leukemia. ASH Annual Meeting Abstracts, 114, 790.

[13] Ravandi, F., Estey, E.H., Appelbaum, F.R., Lo-Coco, F., Schiffer, C.A., Larson, R.A., Burnett, A.K. and Kantarjian, H.M. (2012) Gemtuzumab Ozogamicin: Time to Resurrect? Journal of Clinical Oncology, 30, 3921-3923.

[14] Rowe, J.M. and Lowenberg, B. (2013) Gemtuzumab Ozogamicin in Acute Myeloid Leukemia: A Remarkable Saga about an Active Drug. Blood, 121, 4838-4841.

[15] Burnett, A.K., Hills, R.K., Milligan, D., Kjeldsen, L., Kell, J., Russell, N.H., Yin, J.A., Hunter, A., Goldstone, A.H. and Wheatley, K. (2011) Identification of Patients with Acute Myeloblastic Leukemia Who Benefit from the Addition of Gemtuzumab Ozogamicin: Results of the mrc aml15 trial. Journal of Clinical Oncology, 29, 369-377.

[16] Castaigne, S., Pautas, C., Terre, C., Raffoux, E., Bordessoule, D., Bastie, J.N., Legrand, O., Thomas, X., Turlure, P., Reman, O., de Revel, T., Gastaud, L., de Gunzburg, N., Contentin, N., Henry, E., Marolleau, J.P., Aljijakli, A., Rousselot, P., Fenaux, P., Preudhomme, C., Chevret, S., Dombret, H., Acute Leukemia French Association (2012) Effect of Gemtuzumab Ozogamicin on Survival of Adult Patients with De-Novo Acute Myeloid Leukaemia (alfa-0701): A Randomised, Open-Label, Phase 3 Study. Lancet, 379, 1508-1516. http://dx.doi.org/10.1016/S0140-6736(12)60485-1

[17] Burnett, A.K., Russell, N.H., Hills, R.K., Kell, J., Freeman, S., Kjeldsen, L., Hunter, A.E., Yin, J., Craddock, C.F., Dufva, I.H., Wheatley, K. and Milligan, D. (2012) Addition of Gemtuzumab Ozogamicin to Induction Chemotherapy Improves Survival in Older Patients with Acute Myeloid Leukemia. Journal of Clinical Oncology, 30, 3924-3931.

[18] Brunnberg, U., Mohr, M., Noppeney, R., Durk, H.A., Sauerland, M.C., Muller-Tidow, C., Krug, U., Koschmieder, S., Kessler, T., Mesters, R.M., Schulz, C., Kosch, M., Buchner, T., Ehninger, G., Duhrsen, U., Serve, H. and Berdel, W.E. (2012) Induction Therapy of aml with ara-c plus Daunorubicin versus ara-c plus Gemtuzumab Ozogamicin: A Randomized Phase ii Trial in Elderly Patients. Annals of Oncology, 23, 990-996. 